\title{
ON PLATEAU'S PROBLEM FOR MINIMAL SURFACES OF HIGHER GENUS IN $\mathbf{R}^{3}$
}

\author{
BY FRIEDRICH TOMI AND ANTHONY TROMBA
}

The classical solution of the Plateau problem by Radó [10] and Douglas [3] shows that any rectifiable Jordan curve in $\mathbf{R}^{3}$ is spanned by a minimal surface of disc type. Under what conditions a minimal surface of a given higher genus exists, spanning a given Jordan curve in a Riemannian manifold $N$, seems to be a much more difficult problem. For compact minimal surfaces without boundary and in case $N$ has sufficient topological complexity, the "incompressibility" method of Schoen and Yau gives a sufficient condition for existence.

In [4] Douglas did develop a method to treat the problem of when a given contour is spanned by a surface of genus $\mathfrak{p}$. Douglas' condition, however, seems quite difficult to verify in concrete cases. In this paper we will give simple geometric and topological sufficient conditions.

THEOREM. Let $N$ be a solid torus of class $C^{3}$ and genus $\mathfrak{g}$ in $\mathbf{R}^{3}$ whose boundary has nonnegative inward mean curvature, and let $\gamma \in \Pi_{1}(N)$ denote the homotopy class of a rectifiable Jordan curve $\Gamma$ in $N$.

(a) If $\mathfrak{g}=2 \mathfrak{p}$ and $\gamma=a_{1} a_{2} a_{1}^{-1} a_{2}^{-1} \cdots a_{2 \mathfrak{p}-1} a_{2 \mathfrak{p}} a_{2 \mathfrak{p}-1}^{-1} a_{2 \mathfrak{p}}^{-1}$ where $a_{1}, \ldots, a_{2 \mathfrak{p}}$ is a basis for $\Pi_{1}(N)$ then $\Gamma$ bounds an immersed oriented minimal surface of genus $\mathfrak{p}$.

(b) If $\mathfrak{g}=1$ and $\gamma=2 \alpha$ for some $\alpha \neq 0$ in $\Pi_{1}(N)$ then $\Gamma$ bounds an immersed minimal surface of Möbius type.

We sketch the proof of part (a).

Let $\Gamma$ be a rectifiable contour in a solid $2 \mathfrak{p}$ torus $N \subset \mathbf{R}^{3}, M$ a surface of genus $\mathfrak{p}$ with $\partial M \cong S^{1}$ the unit circle. Further let $\mathcal{N}_{\Gamma}=\{u: M \rightarrow$ $N \mid u: M \rightarrow \Gamma$ monotonically, $\left.u \in H^{1}\left(M, \mathbf{R}^{3}\right) \cap C\left(M, \mathbf{R}^{3}\right)\right\}$. Denote by $\mathcal{M}$ the $C^{\infty}$ Riemannian metrics $g$ on the Schottky double $\hat{M}$ of $M$ such that the natural involution $T: \hat{M} \hookleftarrow$ is an isometry for $g$. Dirichlet's functional

$$
E: \mathcal{M} \times \mathcal{N}_{\Gamma} \rightarrow R
$$

is defined by

$$
E(g, u)=\frac{1}{2} \sum_{i=1}^{3} \int_{M} g(x)\left(\nabla_{g} u^{i}, \nabla_{g} u^{i}\right) d \mu_{g} .
$$

Let $P$ be the space of all $C^{\infty}$ positive functions on $\hat{M}$ which are symmetric and $D_{0}$ those $C^{\infty}$ diffeomorphisms which fix $\partial M \subset \hat{M}$ (as a set) and are homotopic to the identity. The Teichmüller space for $M$ is then defined to be $\tau=(\mathcal{M} / P) / D_{0}$, a finite-dimensional $C^{\infty}$ manifold of dimension

Received by the editors May 13, 1985.

1980 Mathematics Subject Classification. Primary 53A10, 49F10. 
$-3 \chi(M), \chi(M)$ the Euler characteristic of $M$. The conformal invariance of Dirichlet's functional guarantees that $E$ is well defined as a map

$$
E: \tau \times \mathcal{N}_{\Gamma} \rightarrow \mathbf{R} \text {. }
$$

Let $D / D_{0}=\Upsilon$ be the modular group of $M$. Then the Riemann space $R$ of moduli is defined as $R=T / \Upsilon$.

For $u \in \mathcal{N}_{\Gamma}$ consider the introduced map $u_{*}: \pi_{1}(M) \rightarrow \pi_{1}(N)$. Now

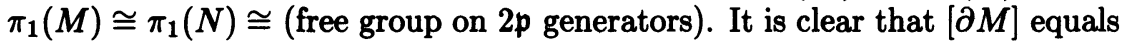
the commutator of the basis of $\pi_{1}(M)$ obtained from the standard polygonal model of $M$. By hypothesis $u_{*}$ then takes the commutator to the commutator. By Zieschang's [11] generalization of a classical result of Dehn (unpublished) and Nielsen [9], $u_{*}$ must be an isomorphism.

Let $\left(\left[g_{n}\right], u_{n}\right) \in \tau \times \mathcal{N}_{\Gamma}$ denote a minimizing sequence. Then since $\left(u_{n}\right)_{*}$ is an isomorphism, $u_{n}$ satisfies the Douglas-Courant nondegeneracy condition. Using the Mumford compactness result for the moduli space and a clever idea of Schoen and Yau [11] one can show that the class of $g_{n}$ in $R$ has a convergent subsequence. This means that there exists a sequence $f_{n} \in D$ such that the pull back $f_{n}^{*}\left(g_{n}\right)$ converges in $\mathcal{M}$. The Courant-Lebesgue Lemma and nondegeneracy show that $u_{n}$ has a convergent subsequence. Lower semicontinuity of $E$ then guarantees the existence of a minimum $([\bar{g}], \bar{u}) \in \tau \times \mathcal{N}_{\Gamma}$ for Dirichlet's functional. One must now show that $([\bar{g}], \bar{u})$ represents a minimal surface. This is not straightforward since we are minimizing Dirichlet's functional subject to an obstacle restraint. Nevertheless this can be done using a regularity result for variational inequalities of the first author [12] and a suitable version of a maximal principle which shows that $\bar{u}$ maps $\stackrel{\circ}{M}$ into $\stackrel{\circ}{N}$. The results of Gulliver, Osserman, Royden $[5,6]$ now imply that the resulting minimal surface is immersed.

\section{REFERENCES}

1. Richard Courant, The existence of minimal surfaces of given topological type, Acta Math. 72 (1940), 51-98.

2. Richard Courant and Herbert Robbins, What is mathematics?, Oxford Univ. Press, New York, 1941.

3. Jesse Douglas, Solution to the problem of Platear, Trans. Amer. Math. Soc. 33 (1931), 263-321.

4. __ Minimal surfaces of higher topological structure, Ann. of Math. (2) 40 (1939), 205298.

5. R. Gulliver, R. Ossermer and H. L. Royden, A theory of branched immersions, Amer. J. Math. 95 (1973), 750-811.

6. R. Gulliver, Branched immersions of surfaces and reduction of topological type. II, Math. Ann. 230 (1977), 23-48.

7. Bob Hardt and Leon Simon, Boundary regularity and embedded solutions for the oriented Plateau problem, Ann. of Math. (2) 110 (1979), 439-486.

8. Stefan Hildebrandt, Boundary behavior of minimal surfaces, Arch. Rational Mech. Anal. 35 (1969), 47-81.

9. J. Nielsen, Die Isomorphiomen der allgemeinen unendlichen Gruppe mit zwei Erzeugenden, Math. Ann. 78 (1918), 385-397. (1933).

10. Tibor Rado, On the Problem of Plateau, Ergeb. Math. Grenzgeb., Springer-Verlag, 
11. Rick Schoen and S. T. Yau, Eacistence of incompressible minimal surfaces and the topology of three manifolds with nonnegative scalar curvature, Ann. of Math. (2) 110 (1979), 127-142.

12. Friedrich Tomi, Variationsprobleme vom Dirichlet-Typ mit einer Ungleichung als Nebenbedingung, Math. Z. 128 (1972), 43-74.

13. Heiner Zieschang, Alternierende Produkte in freien Gruppen, Abh. Math. Sem. Univ. Hamburg 27 (1964), 12-31.

14. Luc Lemaire, Boundary value problems for harmonic and minimal maps of surfaces into manifolds, Ann. Scuola Norm. Sup. Pisa Cl. Sci. (4) 9 (1982), 91-103.

15. William H. Meeks, Uniqueness theorems for minimal surfaces, Illinois J. Math. 25 (1981), 318-336.

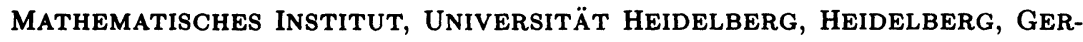
MANY

DePARTMENT OF MATHEMATICS, UNIVERSity OF CAlifornia, SANTA CRUZ, CALIFORNIA 95064 
